\title{
Design and Implementation of Multi-Rate Broadcast Based Link Quality Measurement for Wireless Mesh Network
}

\author{
Seung-Chur Yang, Hyung-Yoon Seo, and Jong-Deok Kim
}

\begin{abstract}
We propose MBAP (Multi-rate Broadcast Active Probing) technique to get the right measurements for link quality in Wireless Mesh Network (WMN). Most routing protocols for WMN make use of link quality-aware routing metrics, such as ETX(Expected Transmission Count) and ETT(Expected Transmission Time), while the hop count is usually used in MANET (Mobile Ad-hoc NETwork). A broadcast based active proving technique is adopted in the previous studies to get the ETX or ETT of a link. However this technique does not reflect the multi-rate feature of WLAN because it uses a single fixed transmission rate for broadcast which usually differs from the actual rate used in data transmissions. MBAP overcomes this shortage by exploiting various rate broadcast frames for probing. We implement MBAP on linux system by modifying WLAN driver and related kernel sub-systems. Experimental results show that MBAP can capture link quality more accurately than the existing techniques.
\end{abstract}

Index Terms-Wireless mesh network, link quality measurement, broadcast active probing, unicast active probing, expected transmission count, expected transmission time.

\section{INTRODUCTION}

The Wireless Mesh Network (WMN) is a multihop wireless network consists of wireless backbone links to provide access point connection [1]. It is a lot of benefit of cost-effective network construction, extensive network coverage and path reliability. WMN is similar to Mobile Ad-hoc Network (MANET) in terms of multihop communication. However, WMN is required high throughput and high reliability than MANET. The hop count is commonly adopted to select a path in MANET, while various link metrics are adopted in WMN [2].

The wireless link metrics, which is widely used in WMN, are Expected Transmission Count (ETX) and Expected Transmission Time (ETT) [3]-[5]. Each of them is based on Packet Delivery Ratio (PDR). Fig. 1 presents the relationship between these methods. The path metric is defined as the sum of each metric, such as Cumulated ETX (CETX) and Cumulated ETT (CETT). According to IEEE 802.11s standard, the path metric is defined as "Airtime link metric", which is similar to CETT. The routing protocol is closely related to path performance. In performance, the wireless link metric is the determining factor. The proposed measurement

Manuscript received November10, 2012; revised January 28, 2013.

The authors are with the Pusan National University, Pusan, 609-735, Republic of Korea (e-mail: elleysc@pusan.ac.kr, tanyak@pusan.ac.kr, kimjd@pusan.ac.kr). method may not be dependant to routing protocol. This system architecture separated by routing protocol and link metric is adopted in IEEE 802.11s standard [6].

Let us suppose that each node knows all link quality in network topology through certain routing protocols. When node $\mathrm{S}$ transmits frames to node $\mathrm{D}$ in figure 1 , the routing protocol must select a path among path S-A-D and path S-B-D. In this case, CETX and CETT of two paths are same 2.1 and 2745 us respectively. Therefore, the routing protocol may select a path of two paths. However, we found out that two paths performance is different in real experiment. We measured throughput for two paths using iperf [7]. Table I presents the PDR and throughput for each path. As you can see, existing wireless link metric does not reflect throughput The reason that these metrics does not reflect link quality effectively is not the metric, but implements issue. According to draft standard, implementing the link metric is out of standard [6].

TABLE I: THE ACCURATE LINK QUALITY MEASUREMENT; THERE IS NO CORRELATION BETWEEN PDR AND THROUGHPUT

\begin{tabular}{cccc}
\hline \hline Path & Link 1 PDR & Link 2 PDR & Throughput \\
\hline S-A-D & S-A: 0.97 & A-D: 0.99 & $33.2 \mathrm{Mbps}$ \\
S-B-D & S-B: 0.98 & B-D: 0.99 & 17.6 Mbps \\
\hline \hline
\end{tabular}

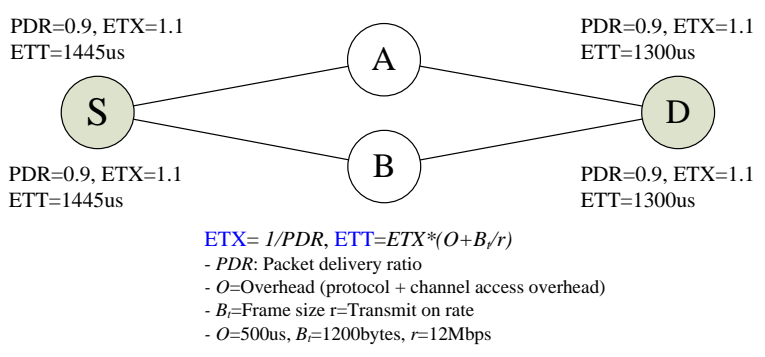

Fig. 1. The relationship between PDR and ETX and ETT.

The existing methods for link quality measurement are mostly based on Broadcast Active Probing (BAP) [2]-[5]. In BAP, nodes send a broadcast frame periodically. Then, receiving nodes measure the link quality. It has the advantage of cost-effectiveness. Broadcast frames are sent by only default bit-rate, while IEEE 802.11 wireless LAN supports multiple bit-rates at the physical layer. The default bit-rate is set to $1 \mathrm{Mbps}$ according to $802.11 \mathrm{~b} / \mathrm{g}$ radio. This approach may cause unexpected throughput, since the measurement bit-rate and data transmission bit-rate is different.

The necessity of using multiple bit-rates is already suggested in [8], [9]. In order to solve this problem, Unicast Active Probing (UAP) is used to measure link quality. This method increases the measurement overhead than BAP, since 
the additional proving frames are required per each link. Moreover, it is hard to measure link quality accurately, because UAP does not consider retransmission process at data-link layer.

This paper present a new method of link quality measurement, called Multi-rate Broadcast based Active Probing (MBAP). Multiple bit-rates is used to measure link quality of a link. This method may accurately measure link quality, but measurement overhead increases. To reduce overhead, we present two schemes to select a set of available bit-rate. It is hard to implement this method, since the modification of device driver is needed at the kernel level programming. The proposed MBAP is implemented in embedded Linux system. We build an outdoor test bed and compare MBAP with existing method such as BAP and UAP. As a result, we perform the effectiveness of MBAP for measurement accuracy.

\section{RELATED WORKS}

The measurement methods for wireless link quality are classified into active and passive method. The active method sends a management frame periodically. The receiving nodes observe and collect transmission status. The passive method observes and collects transmission status by data frames which are generated by users [9]. It is the most efficient and accurate since it uses actual data traffic. However, it also incurs the overhead of probing idle links. Its method does not exist management overhead, and the hybrid method combined of active and passive is mentioned by [8].

As mentioned above, a common method used to measure link quality is active method. The active method is classified into BAP and UAP by a type of transmissions. BAP was originally designed to be aware of link asymmetry. It can measure multiple link quality by one frame, and then measurement overhead is lower than UAP. But, BAP does not reflect multi-rate feature of WLAN [10], [11].

A layer which implements measurement methods is important part in analyzing and understanding wireless link quality. The related works for link quality are mainly implemented by network layer. This approach is easy to implement, but has a measurement limitation in using the element dependant on data link layer. A need for these elements is recognized in single bit-rate transmission in BAP. The unicast transmission is sent by available bit-rate which is determined to bit-rate selection algorithms such as Sample, ONOE and AMRR. In comparison, broadcast frames are sent by the lowest bit-rate in BAP. The available bit-rates of each link are needed to calculate ETT. To achieve this, measurement methods may use APIs to obtain the elements dependant on data link layer.

Although UAP is used to multiple bit-rate transmission, the method can not reflect retransmission process at data link layer. As a result, it is hard to measure wireless link quality accurately. Fig. 2 presents retransmission process at data link layer. When sender sends probe frames by unicast transmission, a sender waits for an acknowledgement of a probe frame. During this time retransmission may occur according to wireless environments. UAP, however, cannot detect any number of retransmission.

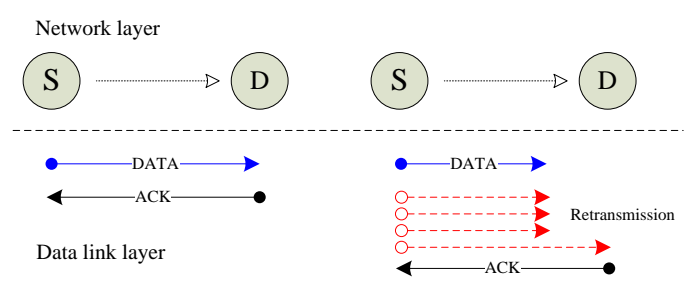

(A)

(B)

Fig. 2. Retransmission process at data link layer and limits of UAP. (A) UAP cannot detect any number of retransmission by measurement, (B) Unicast transmission.

Another limitation of UAP is that link asymmetric is not detected though measurement. In BAP, each node sends and receives probing frame in both directions, so that this method can measures PDR of each link. If transmission failure may occur in an asymmetric link environment, UAP cannot detect which direction happen failure of frames.

In summary, we assume that measurement method considers the element dependant on data link layer. If not, measurement accuracy is unreliable due to multiple bit-rate and link asymmetric. The Madwifi device driver based on Atheros chipset is known as open development standard in research area [12]. In terms of implementation issue, this driver may collect transmission counts per interface, cannot collect transmission counts per link. Therefore, UAP is not suitable to link metric, even though implementation is operated at data link layer. This paper presents measurement method based on BAP with for UAP limitation such as multiple bit-rate and retransmission process.

\section{DESIGN AND IMPLEMENTATION OF MUlti-RATE BROADCAST BASED LINK QUALITY MEASUREMENT}

\section{A. MBAP System Architecture}
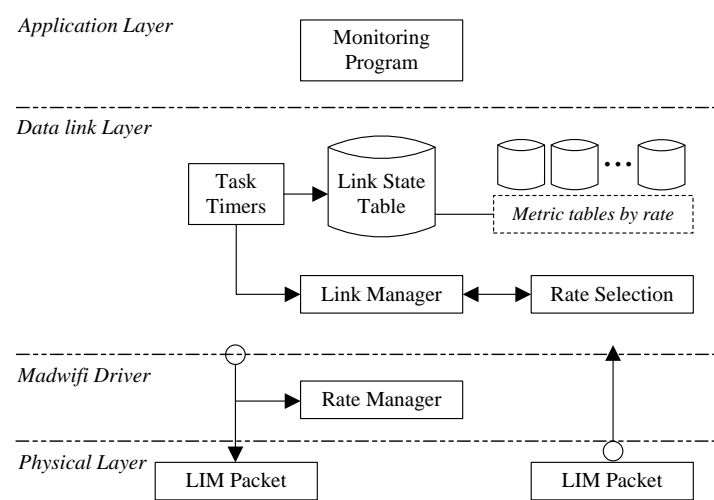

Fig. 3. MBAP system architecture. It consists of a application at application layer and a module at data link layer and modified device driver. The module can load in Linux kernel.

Fig. 3 presents MBAP system architecture. This system uses additional management frame denoted by Link Information Management (LIM). System component is the following.

1) Link State Table: Database contains link quality information.

2) Link Manager: It controls the periodical transmission of LIM and measures link quality and stores Link State Table. 
3) Rate Selection: In order to reduce overhead, it selects a set of available bit-rate.

4) Task Timer: It controls interval of LIM transmission and of calculation of link metrics such PDR.

5) Rate Manager: In Madwifi driver, the bit-rate of LIM frame is controlled by the pre-defined roles.

In Madwifi driver, unicast frames is controlled by bit-rate selection algorithms such as Sample, AMRR, ONOE, and broadcast frames is controlled by the constant variable of mcast_rate. Link Manager stores a selected bit-rate into Control Buffer (CB), which is the field of Socket Buffer. The function of ath_tx_start maps mcast_rate onto a bit-rate of CB. This function is called before data is sent to Hardware Abstraction Layer (HAL), controls bitl-rates.

\section{B. Transmission Rate Selection to Reduce MBAP Overhead}

Table II present transmission bit-rate supported in IEEE $802.11 \mathrm{a} / \mathrm{b} / \mathrm{g}$. If MBAP measures link quality in all of supported bit-rate, the overhead is bigger than BAP. Let us suppose that MBAP periodically sends 1000bytes frame through all of supported bit-rate in IEEE 802.11a, 10 times per second. In the case of BAP, the medium is occupied for $13.3 \mathrm{~ms}(=10 \times 1000 \times 8 / 6 \mathrm{Mbps})$. If MBAP sends frames through 8 times bit-rate from $6 \mathrm{Mbps}$ to $54 \mathrm{Mbps}$, the medium is occupied for $42.0 \mathrm{~ms}$. The occupied time of MBAP is 3 times higher than BAP.

TABLE II: All Data RATES SPECIFIED IN THE IEEE 802.11A/B/G STANDARD

\begin{tabular}{cc}
\hline \hline Radio & Supported data rate $(\mathrm{Mbps})$ \\
\hline $802.11 \mathrm{a}$ & $6,9,12,18,24,36,48,54$ \\
$802.11 \mathrm{~b}$ & $1,2,5.5,11$ \\
$802.11 \mathrm{~g}$ & $1,2,5.5,6,9,12,18,24,36,48,54$ \\
\hline \hline
\end{tabular}

In order to support multi-rate, the increase of measurement overhead is essential to MBAP. To solve this problem, we exclude the transmission bit-rates which are less likely to be sent. The Signal-to-Noise Ratio (SNR) and auto transmission bit-rate algorithm is used to exclude some bit-rates.

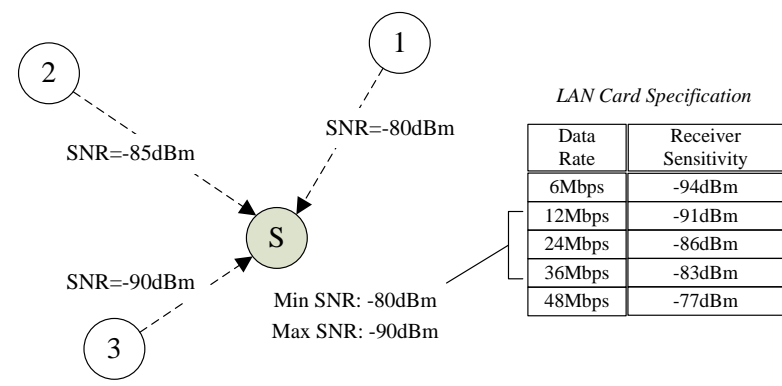

Fig. 4. The multi-rate selection method using SNR. Receiver sensitivity dependant on wireless NIC is used.

First, we adopt receiver sensitivity of wireless network interface card to select a set of available bit-rate. Atheros chipset define the receiver sensitivity in the following Fig. 4. MBAP measure the Receiver Strength Signal Indicator (RSSI) of frame which is sent to neighbor nodes, and stores Link State Table. We predict the available bit-rates when compared to RSSI and receiver sensitivity.

Let us suppose that there are node N1, node N2 and node
$\mathrm{N} 3$ around node $\mathrm{S}$ as shown to Fig. 4. Each SNR is $-80 \mathrm{dBm}$, $-85 \mathrm{dBm}$ and $-90 \mathrm{dBm}$. According to receiver sensitivity table, node $\mathrm{S}$ can sends data frames to $36 \mathrm{Mbps}, 24 \mathrm{Mbps}$ and $12 \mathrm{Mbps}$, respectively. Therefore, we may exclude bit-rates over upper-bound, 48Mbps and 54Mbps, under lower-bound, $6 \mathrm{Mbps}$. As a result, measurement overhead has drastically decreased.

In some situation, unexpected situations may sometimes happen, for example actual transmission bit-rate is not enough to predict bit rate based on RSSI. The reason why auto transmission bit rate algorithms selects a bit-rate not by RSSI, but by transmission status such continuous PDR [8]. The bit-rates obtained by auto transmission bit-rate algorithms are reasonable to accurately reflect link quality in various wireless environments.

We modified wireless device driver that enables to store bit-rate of each link at data link layer in Link State Table. It decrease MBAP measurement overhead by generated only a set of bit rate.

\section{EXPERIMENT AND ANALYSIS}

\section{A. Experiment Scenario}

The purpose of experiments can be divided into three types. First, MBAP effectively reflects the wireless environment regardless of a layer, compared to BAP and UAP. Second, we show the asymmetric wireless link. Final, MBAP accurately measures link quality.

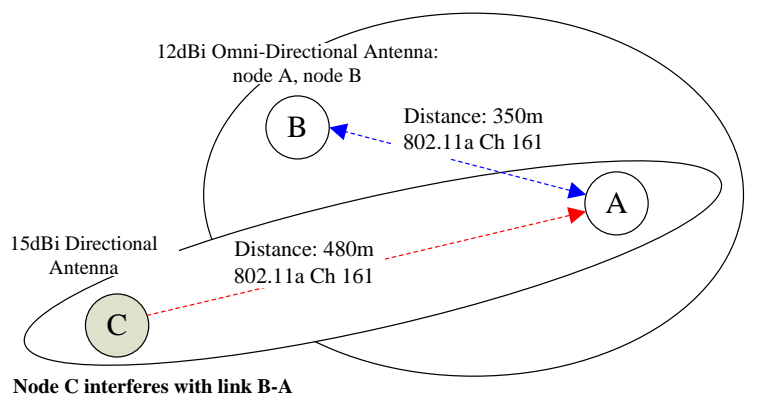

Fig. 5. The experimental network topology.

In order to evaluate these performances, we build a outdoor test bed which consists of three wireless mesh router as shown in figure 5. The mesh router is used in ALIX 3c2 boards with an AMD Geode processor, 2 minipci slots. Each mesh router is loaded in Pyramid Linux kernel 2.6.19 and madwifi 0.9.4. The channel of wireless interface card is set to a 161 channel in IEEE 802.11a to avoid adjacent interference.

We adopt various antennas type for the purpose of an experiment. The $12 \mathrm{dBi}$ omni-directioal antenna is attached in node $\mathrm{A}$ and node $\mathrm{B}$. The $15 \mathrm{dBi}$ directional antenna is attached in node C. In this way, we can confirm the link asymmetric and the link quality due to hidden terminal problem for node $\mathrm{C}$.

\section{B. Analysis of Experimental Results}

Fig. 6 presents the PDR of link A-B.BAP measured by BAP, UAP and MBAP. Fig. 7 presents the PDR of link B-A measured by BAP, UAP and MBAP. Each experiment run 
for 30 minutes and the PDR is measured every one minutes. The PDR of link A-B is measured from node B through the frames is sent to node A. In Fig. 7, MBAP is represented with multiple links for each bit rate, while BAP and UAP are represented with single link.

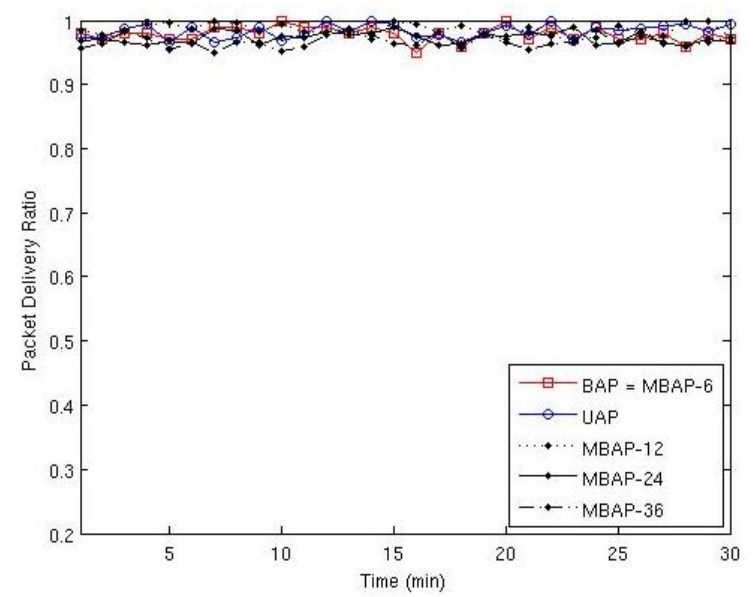

Fig. 6. The PDR of BAP, UAP and MBAP for link A-B. This PDR is measured from node $B$, and is approximately 1 . SNR of frames which is sent by node $\mathrm{A}$ is enough to select $36 \mathrm{Mbps}$ bit-rate.

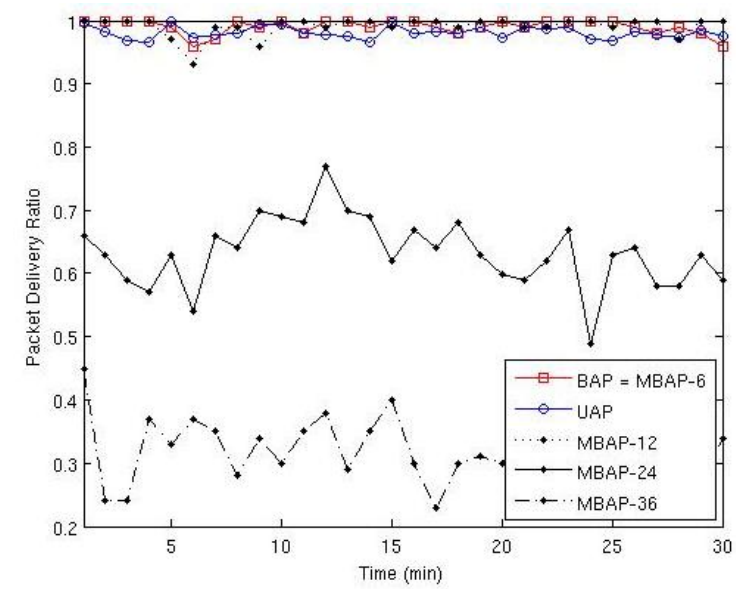

Fig. 7. The PDR of BAP, UAP and MBAP for link B-A. This PDR is measured from node $\mathrm{A}$. We assume that node $\mathrm{C}$ may interfere in the path between node $\mathrm{A}$ and node $\mathrm{B}$.

In BAP, the PDR of link A-B and link B-A are same to one. The PDR of link B-A decreases dramatically over $24 \mathrm{Mbps}$ bit rate in MBAP as shown in Fig. 7. If node B sends data frames to node A over 24Mbps, the packet loss and retransmission happen in communication. As a result, BAP is not easy to reflect multi-rate support and to detect link asymmetric.

In UAP, the PDR of link A-B and link B-A are also same to one. Therefore, it cannot detect link asymmetric. However, UAP sends probe frames by unicast transmission to support multi-rate. We had been captured unicast frames with Omnipeek, which is a packet analyzer software tool from WildPackets. As a result, the bit rate of unicast frame was 24Mbps. An important of this analysis, the PDR of UAP is higher than MBAP-24, since UAP contains retransmission count. UAP has a limitation of accurate measurement due to retransmission process at data link layer, while it can accurately measure link quality in unicast transmission.

In comparison, MBAP may detect link asymmetric even though probe frames are broadcast. Of course, we reduce measurement overhead by the same level of BAP, as mentioned in Section III.

We had been experiment to verify that MBAP accurately reflect real-time wireless environment and precisely predict link metrics. To do so, we measure Round Trip Time (RTT) between node $\mathrm{A}$ and node $\mathrm{B}$ using ping program. The node $\mathrm{A}$ sends 1500 bytes ICMP messages to node B periodically. We generate interference with traffic which is sent to node $\mathrm{C}, 10$ minutes and 20 minutes for 5 miniute, after an experiment starts.

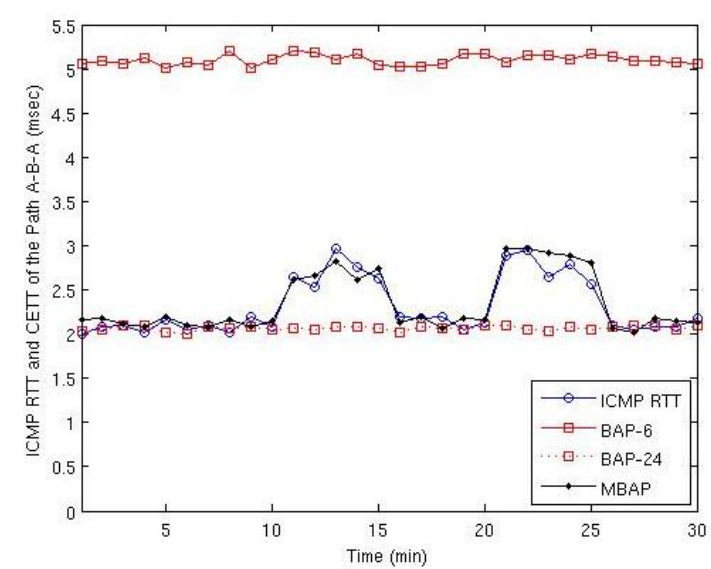

Fig. 8. A comparison with CETT of MBAP and RTT. RTT of MBAP is calculated as the sum of ETT for a path A-B-A, and bit-rate of the lowest PDR is used to calculate ETT of each link.

In MBAP, we calculated ETT of link A-B and link B-A, then calculated CETT for a path A-B-A. For comparison, we also calculate CETT using BAP. In order to lead ETT for BAP, we try to send probe frames over 6Mbps (BAP-6) and $24 \mathrm{Mbps}$ (BAP-24) respectively. Figure 8 presents MBAP reflect various wireless environment effectively.

\section{CONCLUSION}

The accurate measurement of link quality is essential to provide high through and high reliability in WMN. Nevertheless, related works such as BAP and UAP cannot support multi-rate, link asymmetric and retransmission at data link layer. So these methods to measure link quality have limits of accuracy measurement for link metric.

This paper proposes a measurement method, called MBAP, to support multi-rate broadcast for WMNs. The main idea of MBAP is simple, but it is hard to implement in real system. The modification of wireless device driver is needed on very low-level of kernel. The accuracy of the proposed scheme is higher, but overhead can be increased. To solve a problem, we present a method of bit-rate selection using SNR and auto transmission bit-rate algorithms. So MBAP can reduce measurement overhead by the same level of BAP.

We implement MBAP on Linux system by modifying WLAN driver and related kernel sub-systems. Experimental results show that MBAP can capture link quality more accurately than the existing techniques.

\section{ACKNOWLEDGMENT}

This research was supported by Basic Science Research Program through the National Research Foundation of Korea 
(NRF) funded by the Ministry of Education, Science and Technology (2012-0001578).

\section{REFERENCES}

[1] I. F. Akyildiz and X. Wang, "A survey on wireless mesh networks," IEEE Communications Magazine, vol. 43, no. 9, pp. 23-30, Sept. 2005.

[2] C. E. Koksal and H. Balakrishnan, "Quality-Aware routing metrics for time-varying wireless mesh networks," IEEE JSAC, vol. 24, no. 11, pp. 1984-94, Nov. 2006

[3] R. Draves, J. Padhye, and B. Zill, "Routing in multi-radio, multi-hop wireless mesh networks," in Proc. ACM MobiCom, Sep. 2004, pp. $114-28$.

[4] D. S. D. Couto, D. Aguayo, J. Bicket, and R. Morris. "A high-throughput path metric for multi-hop wireless routing," in Proc. ACM MobiCom, San Diego, CA, Sep. 2003.

[5] D. Passos et al., "Mesh network performance measurements," in Proc. ISMICT, Dec. 2006.

[6] IEEE 802.11s/D1.06, Wireless LAN MAC and PHY specification: Amendment, Mesh Networking, July 2007.

[7] Iperf: The TCP/UDP Bandwidth Measurement Tool. [Online]. Available: http://www.dast.nlanr. net/Projects/Iperf/.

[8] K. S. K. Kim, "On accurate measurement of link quality in multi-hop wireless mesh networks," in Proc. ACM Mobicom, Sep. 2006, pp. $38-49$.

[9] M. Lacage, M. Manshaei, and T. Turletti, "IEEE 802.11 Rate Adaptation: APractical Approach," in Proc. MSWiM, Oct. 2004.

[10] L. Verma, S. Kim, S. Choi, and S. Lee,"Reliable, low overhead link quality estimation for 802.11 wireless mesh networks," in Proc. SECON, June 2008.

[11] A. Kashyap, S. Ganguly, and S. R.Das, "A measurement based approach to modelling link capacity in 802.11-based wireless networks," in Proc. ACM MobiCom, Sep. 2007.

[12] MadWiFi: Multiband Atheros Driver for WiFi. [Online]. Available: http://www.madwifi.org/.

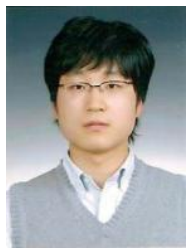

Seung-Chur Yang received his B.S. degrees in Computer Science Engineering (CSE) from Catholic University of Pusan in 2006, and received the M.S. degrees in CSE from Pusan National University in 2008. He is now a Ph.D. candidate of CSE in Pusan National University. His current research interests include Wireless Mesh Networks and mobility support in wireless networks.

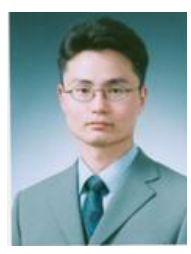

Hyung-Yoon Seo received the B.S. degree in Computer Information Engineering from Hanseo University, Korea in 2002 and received the M.S. degrees in Computer Science and Engineering from Pusan National University, Korea in 2011. He is now a Ph.D. candidate of Computer Science and Engineering in Pusan National University. His current research interests include Mobile Multimedia Communication Systems, MAC and Routing Protocols from Mobile Wireless Networks, and All-IP based Network Convergence.

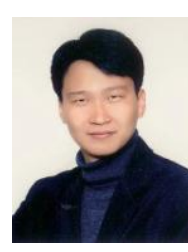

Jong-Deok Kim received his B.S. M.S. degrees in Computer Science from Seoul National University in 1994 and 1996, respectively, his Ph. D. in Computer Science and Engineering from Seoul National University in 2003. He joined Telcoware in 2003, which is a Korea venture company focusing on wireless core network. $\mathrm{He}$ is now an Associate Professor of Computer Science and Engineering in Pusan National University since 2006. His current research interests include RFID/USN, quality of services and mobility support and open service architecture in wireless networks. 